\title{
The importance of electrical mapping of VT in the approaching era of clinical imaging
}

Paolo Della Bella ${ }^{1}$ and Antonio Frontera ${ }^{1}$

${ }^{1}$ San Raffaele Hospital

June 10, 2020

\begin{abstract}
We are facing a new challenge: will imaging be the resolutive tool or will new mapping catheters and mapping system together with mathematical simulation solve this rebus? Clinical imaging will always remain attractive, particularly for elective cases and may add decisive information to best plan an ablation strategy: it represents a great tool in the hands of the electrophysiologist; however, as electrophysiologists, the imaging we should pursue is electrical - the depiction of the entire reentry circuit remains the sole proof of the target to ablate.
\end{abstract}

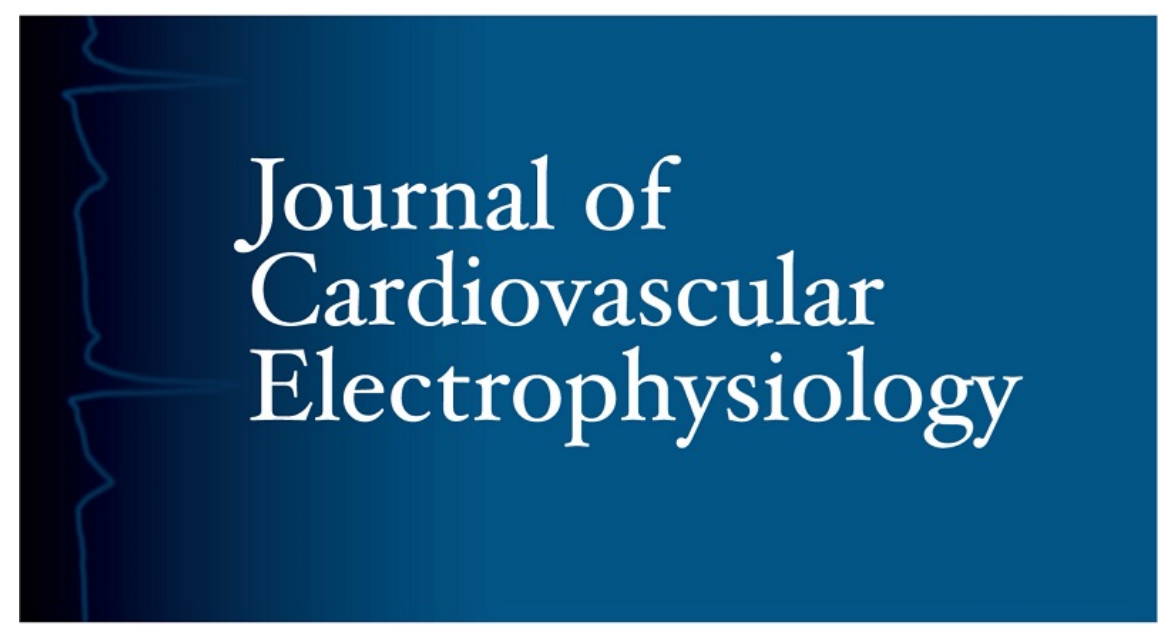

Editorial by Antonio Frontera and Paolo Della Bella

Arrhythmology Department, IRCCS San Raffale, Milan, Italy 
Looking back over the years, the approach to ventricular tachycardia ablation has changed significantly. Initial efforts began in the 1980's consisted of intra-operative mapping performed during cardiac surgery with 20-electrode arrays placed by hand on the endo-epicardial surface(1): this was the first electrical imaging and was subsequently used to guide subendocardial resection. Approaches then transitioned to catheterbased techniques in the 1990s when direct current shock energy and radiofrequency energy were used to modify abnormal areas responsible for the genesis of ventricular arrhythmias(2)(3). Later, a significant transformative step forward was the advent of 3D mapping systems which allowed for detailed activation mapping and the targeting of pathological substrate.

However, the cornerstone of electrophysiological mapping has been the development of multi-spline, multi electrode mapping tools which has elevated the concept of high density mapping to prominence. Today, 3D maps created during sinus rhythm provide excellent substrate characterization $(4)(*)$ : LAVA and LPs became our main targets as expressions of channels and slow conduction areas(5)(6). Furthermore, due to multi electrode mapping catheters, even mapping of hemodynamically non tolerated VT has become feasible: the enhanced recording density and sweeping characteristics that cover a large area in a short amount of time allow for the electroanatomic imaging of the diastolic pathway of the VT reentrant circuit (7).

In the last decade, technological advances in pre-procedural imaging(8) has allowed for the merging of cardiac MRI to 3D mapping systems adding crucial information such as ventricular thickness(9), scar dimensions, and coronary artery localization. The role of cardiac imaging has shifted from a diagnostic to an adjunctive tool to guide our interventional approaches for the treatment of VT, particularly in the setting of post-myocardial infarction VT.

In this issue of Journal of Cardiovascular Electrophysiology, Nazarian et al (reference) published first evidence of specific features of electroanatomic maps with LGE voltage mapping and have highlighted the importance of cardiac magnetic resonance as an adjunct for characterizing the VT substrate in the setting of non ischemic cardiomyopathy (NICM). The authors have added another piece to the NICM puzzle: clinical imaging may help the electrophysiologist look transmurally. In fact, the authors state that late gadolinium enhanced (LGE) distribution predicts the epicardial distribution of scar in electroanatomic maps. EGMs are expressions of signal propagation, and any delay or obstacle found along the trajectory results in longer duration or fractionation. However, current mapping only takes into account the planar propagation of the impulse and not the transmurality of the scar. Nazarian et colleagues elegantly demonstrate what we suspected: local activity signal abnormalities (LAVA) and delayed activity (LPs) were more prevalent in non transmural (mid-myocardial and subepicardial) LGE locations: this confirms that current mapping technology can still detect, in non-transmural scar, signal abnormalities. Indeed, sites with transmural LGE were associated with longer duration EGMs and fractionated potentials: this increased duration is an expression of delayed propagation in this deep substrate. However, it is clear we are missing electrical information: clinical imaging may fill this void.

The battlefield is in the depth of the myocardium where signal propagates in three dimensions where we are unable to map. This research opens new scenarios in the setting of VT ablation in NICM patients: the first step of clinical imaging in the 3D characterization of deep substrate.

Based on our experience(10)(11), as well as reflected in the literature(12), NICM poses a difficult substrate in which the outcome, freedom from ventricular arrhythmias, remains lower as compared to ischemic cardiomyopathy.

Recently, Tung et colleagues(13) demonstrated via simultaneous endocardial and epicardial mapping that VT circuits often involve the 3-dimensions; in this research, the authors documented that in NICM patients, $28 \%$ of cases had the reentry circuit exhibiting planar propagation and complete reentry confined to the endocardium. 3D circuitry occurred in $49 \%$ of cases. Recently, our group demonstrated that during VT, the complete electroanatomic imaging of diastolic pathway not only allows for targeted ablation of all areas that are operational during VT, but results in favorable success rates, with up to $88 \%$ freedom from VT out to 18 months (14). Findings such as these only emphasize the importance of accurate and thorough mapping 
of VT circuitry in order to effectively eliminate VT.

We are facing a new challenge: will imaging be the resolutive tool or will new mapping catheters and mapping system together with mathematical simulation solve this rebus?

Clinical imaging will always remain attractive, particularly for elective cases and may add decisive information to best plan an ablation strategy: it represents a great tool in the hands of the electrophysiologist; however, as electrophysiologists, the imaging we should pursue is electrical - the depiction of the entire reentry circuit remains the sole proof of the target to ablate.

In conclusion, let cardiac imaging play an integral role in determining the underlying etiology and prognostic significance of VT, but let electrophysiologists perform the electrical imaging they were deemed to.

\section{REFERENCES}

1. Kienzle MG, Miller J, Falcone RA, Harken A, Josephson ME. Intraoperative endocardial mapping during sinus rhythm: relationship to site of origin of ventricular tachycardia. Circulation. 1984 Dec;70(6):957-65.

2. Klein LS, Shih HT, Hackett FK, Zipes DP, Miles WM. Radiofrequency catheter ablation of ventricular tachycardia in patients without structural heart disease. Circulation. 1992 May;85(5):1666-74.

3. Gonska BD, Cao K, Schaumann A, Dorszewski A, von zur Mühlen F, Kreuzer H. Catheter ablation of ventricular tachycardia in 136 patients with coronary artery disease: results and long-term follow-up. J Am Coll Cardiol. 1994 Nov 15;24(6):1506-14.

4. Martin R, Maury P, Bisceglia C, Wong T, Estner H, Meyer C, et al. Characteristics of Scar-Related Ventricular Tachycardia Circuits Using Ultra-High-Density Mapping: A Multi-Center Study. Circ Arrhythm Electrophysiol. 2018;11(10):e006569.

5. Vergara P, Trevisi N, Ricco A, Petracca F, Baratto F, Cireddu M, et al. Late potentials abolition as an additional technique for reduction of arrhythmia recurrence in scar related ventricular tachycardia ablation. J Cardiovasc Electrophysiol. 2012 Jun;23(6):621-7.

6. Frontera, Melillo F, Baldetti L, High-Density Characterization of the Ventricular Electrical Substrate During Sinus Rhythm in Post-Myocardial Infarction Patients. J Am Coll Cardiol Electrophysiology. 2020 June 13 (in press)

7. Okubo K, Frontera A, Bisceglia C, Paglino G, Radinovic A, Foppoli L, et al. Grid Mapping Catheter for Ventricular Tachycardia Ablation. Circ Arrhythm Electrophysiol. 2019;12(9):e007500.

8. Hooks DA, Berte B, Yamashita S, Mahida S, Sellal J-M, Aljefairi N, et al. New strategies for ventricular tachycardia and ventricular fibrillation ablation. Expert Rev Cardiovasc Ther. 2015 Mar;13(3):263-76.

9. Takigawa M, Duchateau J, Sacher F, Martin R, Vlachos K, Kitamura T, et al. Are wall thickness channels defined by computed tomography predictive of isthmuses of postinfarction ventricular tachycardia? Heart Rhythm. 2019;16(11):1661-8.

10. Okubo K, Gigli L, Della Bella P. Catheter ablation of ventricular tachycardia in nonischemic cardiomyopathy. J Arrhythmia. 2018 Aug;34(4):347-55.

11. Oloriz T, Silberbauer J, Maccabelli G, Mizuno H, Baratto F, Kirubakaran S, et al. Catheter ablation of ventricular arrhythmia in nonischemic cardiomyopathy: anteroseptal versus inferolateral scar sub-types. Circ Arrhythm Electrophysiol. 2014 Jun;7(3):414-23.

12. Hennig A, Salel M, Sacher F, Camaioni C, Sridi S, Denis A, et al. High-resolution three-dimensional late gadolinium-enhanced cardiac magnetic resonance imaging to identify the underlying substrate of ventricular arrhythmia. Eur Eur Pacing Arrhythm Card Electrophysiol J Work Groups Card Pacing Arrhythm Card Cell Electrophysiol Eur Soc Cardiol. 2018 01;20(FI2):f179-91. 
13. Tung R, Raiman M, Liao H, Zhan X, Chung FP, Nagel R, et al. Simultaneous Endocardial and Epicardial Delineation of 3D Reentrant Ventricular Tachycardia. J Am Coll Cardiol. 2020 Mar 3;75(8):884-97.

14. Hadjis A, Frontera A, Limite L, Complete electroanatomic imaging of the diastolic pathway is associated with improved freedom from ventricular tachycardia recurrence. Circ EP July 2020 (in press). 